\title{
Gut endothelial cells - another line of defence
}

New findings published in Science characterize the gut-vascular barrier (GVB), showing that endothelial cells control the passage of antigens and commensal gut microbiota from the intestine into the bloodstream.

The intestinal barrier prevents the passage of gut microbiota into the circulation yet allows nutrients to be absorbed. Bacteria

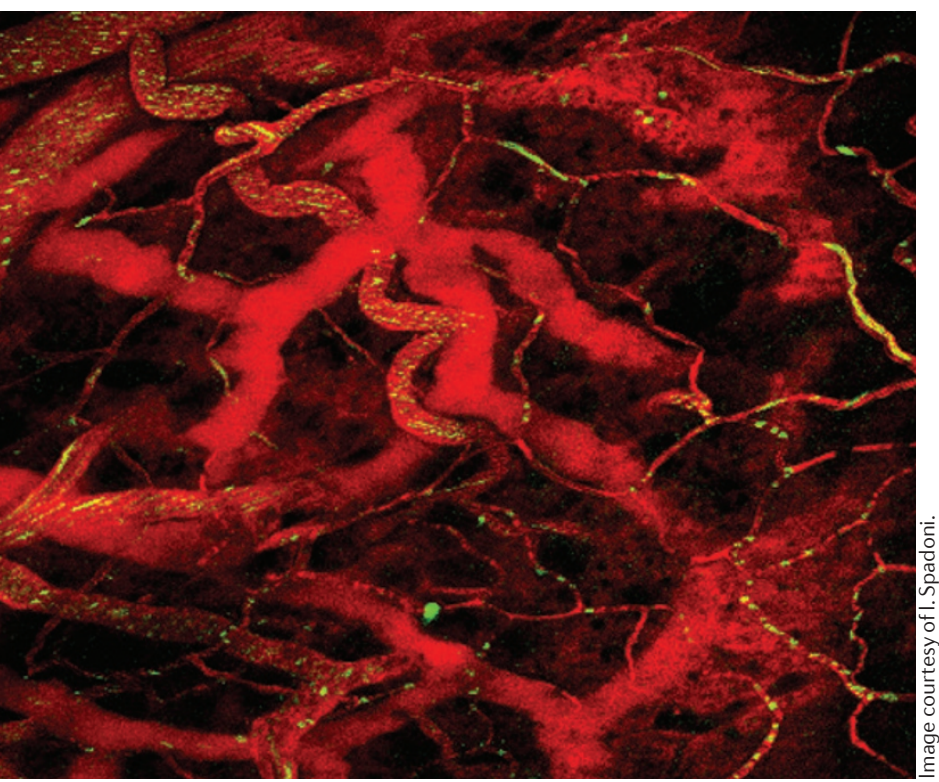

that do manage to cross the intestinal epithelium are prevented from entering the bloodstream, despite the close proximity of blood vessels to epithelial cells. "We wondered why bacteria would not easily enter the blood vessels and reach the liver ... thus, we hypothesized that the endothelial cells of the blood vessels might generate a barrier [to bacteria]," explains corresponding author Maria Rescigno.

Rescigno and colleagues determined the permeability of the GVB in mice by intravenously injecting fluorescently labelled particles of varying molecular weights. The researchers showed that the GVB of healthy mice were impermeable to $70 \mathrm{kDa}$ particles, indicating that bacteria, which are larger, might be physically excluded from the circulation. In common with other vascular barriers, endothelial cells in the GVB expressed a number of tight junction and adherens junction proteins.

To determine if the ability to cross the epithelial barrier is enough for bacteria to cross the GVB, the investigators constructed a strain of Escherichia coli expressing a recombinant protein that enabled it to penetrate the gut epithelium. This bacteria was excluded from the circulation of infected mice despite crossing the epithelial barrier, suggesting that intestinal endothelial cells constitute a distinct vascular barrier against gut microorganisms.

Lastly, the researchers showed that the GVB is present in healthy human intestine by staining for junctional protein expression on intestinal endothelial cells. In intestinal tissue from patients with coeliac disease and no observable epithelial barrier defect, a marker of endothelial permeability was upregulated and was associated with increased liver damage, defined by raised serum alanine aminotransferase levels. "We now want to focus on [endothelial barrier function in] ... diseases that are related to the gut-liver axis and that might be triggered by mucosal barrier defects," concludes Rescigno.

Hugh Thomas

ORIGINAL ARTICLE Spadoni, I. et al. A gut-vascular barrier controls the systemic dissemination of bacteria. Science 350, 830-834 (2015) 